\title{
Second-Line Therapy
}

National Cancer Institute

\section{Source}

National Cancer Institute. Second-Line Therapy. NCI Thesaurus. Code C54350.

The second preferred therapy for a particular condition, used after first-line treatment fails or if a person cannot tolerate first-line drugs. 